\title{
Hydrogen Permeation Performance Comparison among Palladium and Other Membranes with Different Permeation Laws
}

\author{
Shigeki Hara, Alessio Caravella, Misaki Ishitsuka, Hiroyuki Suda, Masakazu Mukaida, Kenji \\ Haraya, Erika Shimano*, Yasuhisa Tanaka*, and Tomoya Tsuji* \\ National Institute of Advanced Industrial Science and Technology (AIST), Central 5, 1-1-1, Higashi, Tsukuba 305-8565, Japan \\ Fax: 81-29-861-4733, e-mail: s.hara@aist.go.jp \\ *College of Industrial Technology, Nihon University, 1-2-1, Izumicho, Narashino 275-8575, Japan,
}

\begin{abstract}
Palladium membranes are useful to produce pure hydrogen by allowing only hydrogen to pass through. However, palladium, a platinum group metal, is limited in resources and expensive. Therefore, alternative membranes are intensively being developed including other metal membranes, porous ceramic membranes, etc. Comparison among them is essential in the development but difficult because these membranes obey different permeation laws. To overcome this difficulty, pressure-dependent permeance is applied to membranes of different thicknesses and different materials: pure palladium membranes $50 \mu \mathrm{m}$ and $200 \mu \mathrm{m}$ thick, a pure niobium membrane, and a silica membrane without pressure dependence in permeance. The result demonstrates that pressure-dependent permeance can describe hydrogen permeation flux more precisely than the conventional square-root law and that it enables comparison among membranes with different thicknesses made of different materials. Suitable operating pressure conditions for each membrane are proposed according to the comparison.

Key words: palladium, hydrogen, membranes, permeability, permeance, square-root law
\end{abstract}

\section{INTRODUCTION}

Palladium membranes are useful to produce pure hydrogen by allowing only hydrogen to pass through. However, palladium, a platinum group metal, is limited in resources and expensive, so that alternative membranes are intensively being developed including other metal membranes, porous ceramic membranes, etc. In the development, comparison among them is essential but these membranes obey different permeation laws.

For metal membranes, hydrogen permeation flux, $J$ $\left[\mathrm{mol} / \mathrm{m}^{2} \mathrm{~s}\right]$, is conventionally described in the following square-root law [1-4]:

$$
J=\frac{\phi}{l}\left(\sqrt{p^{\mathrm{f}}}-\sqrt{p^{\mathrm{p}}}\right),
$$

where $l[\mathrm{~m}], p^{\mathrm{f}}[\mathrm{Pa}]$, and $p^{\mathrm{p}}[\mathrm{Pa}]$ are membrane thickness, feed-side pressure, and permeation-side pressure, respectively. The permeability, $\phi\left[\mathrm{mol} / \mathrm{m} \mathrm{s} \mathrm{Pa}^{0.5}\right]$, is commonly used as a measure indicating permeation ability. However, the square-root permeability is normalized by membrane thickness, so that thin and thick membranes made of the same membrane material have fundamentally the same value. In other words, the effort of decreasing thickness to improve permeation flux is vanished in permeability. Additionally, deviation from the square-root law has been widely reported [3-7]. Although a power law has often been adopted to describe the deviation since 1901 [3-6], power-law's permeability values determined with different powers are incomparable one another.

Meanwhile, permeance is used as a measure indicating membrane performance for palladium-ceramic composite membranes, polymer membranes, ceramic membranes, etc [8]. Permeance, $P$ $\left[\mathrm{mol} / \mathrm{m}^{2} \mathrm{~s} \mathrm{~Pa}\right]$, is defined in the following form:

$$
J=P\left(p^{\mathrm{f}}-p^{\mathrm{p}}\right) .
$$

Because permeance is used for so wide variety of membranes, it seems more useful in terms of membrane comparison than permeability. For dense metal membranes, however, permeation flux is not described well. This is why permeance is not adopted for dense metal membranes.

We have recently proposed pressure-dependent permeability [9]. By introducing pressure dependence into permeability, permeation behavior can be precisely described. Additionally, resultant permeability is comparable for metal membranes even if the permeation behavior obeys neither in the square-root law nor in the power law.

This pressure dependence can be introduced into permeance as well. This procedure is explained in a previous report [10]. The pressure-dependent permeance is expected to realize both precise description and comparison among various membranes.

The aim of this study is to demonstrate this idea: First, the theoretical background $[9,10]$ is briefly explained. Second, the pressure-dependent permeance is applied to various membranes to demonstrate precise description and comparison among them: pure palladium membranes $50 \mu \mathrm{m}$ and $200 \mu \mathrm{m}$ thick measured in our group [10,11], a pure palladium membrane described in the power law by Hurlbert and Konecny [6], a pure niobium membrane reported by Zhang et al. [7], and a 
Table I. Comparison among different measures to estimate permeation flux through metal membranes.

\begin{tabular}{|c|c|c|c|}
\hline & $\begin{array}{l}\text { Square-root law } \\
\text { (Conventional for metal) }\end{array}$ & $\begin{array}{l}\text { Liner law } \\
\text { (Conventional for Silica, } \\
\text { polymer, etc.) }\end{array}$ & $\begin{array}{l}\text { Pressure-dependent measures } \\
\text { (This study) }\end{array}$ \\
\hline $\begin{array}{l}\text { Definition of permeability } \\
\qquad\left[\mathrm{mol} / \mathrm{m} \mathrm{s} \mathrm{Pa}^{0.5}\right]\end{array}$ & $\phi=\frac{l J}{\sqrt{p^{\mathrm{f}}}-\sqrt{p^{\mathrm{p}}}}$ & & $\phi(p)_{p=p^{\mathrm{f}}}=\frac{\mathrm{d}(l J)}{\mathrm{d} \sqrt{p^{\mathrm{f}}}}$ \\
\hline $\begin{array}{l}\text { Flux estimation }\left[\mathrm{mol} / \mathrm{m}^{2} \mathrm{~s}\right]\end{array}$ & $J=\frac{\phi}{l}\left(\sqrt{p^{\mathrm{f}}}-\sqrt{p^{\mathrm{p}}}\right)$ & $J=P\left(p^{\mathrm{f}}-p^{\mathrm{p}}\right)$ & $J=\frac{1}{l} \int_{\sqrt{p^{\mathrm{p}}}}^{\sqrt{p^{\mathrm{f}}}} \phi(p) \mathrm{d} \sqrt{p}=\int_{p^{\mathrm{p}}}^{p^{\mathrm{f}}} P(p) \mathrm{d} p$ \\
\hline $\begin{array}{l}\text { Definition of permeance } \\
\qquad\left[\mathrm{mol} / \mathrm{m}^{2} \mathrm{~s} \mathrm{~Pa}\right]\end{array}$ & & $P=\frac{J}{p^{\mathrm{f}}-p^{\mathrm{p}}}$ & $P(p)_{p=p^{\mathrm{f}}}=\frac{\mathrm{d} J}{\mathrm{~d} p^{\mathrm{f}}}$ \\
\hline
\end{tabular}

silica membrane without pressure dependence in permeance reported by Tsuru et al [8]. Because original experimental data are from the literature, the experiment is explained as short as possible. Finally, hydrogen permeation performance with different permeation laws is compared.

\section{TEORY}

Relation between conventional and pressuredependent measures are summarized in Table I. Square-root permeability is defined by $l J$ divided by square-root pressure difference, whilst the function representing pressure-dependent permeability is determined by differentiating $l J$ in terms of the square-root of feed side pressure. To estimate permeation flux, the following formula is useful [9]:

$$
J=\frac{1}{l} \int_{\sqrt{p^{\mathrm{p}}}}^{\sqrt{p^{\mathrm{f}}}} \phi(p) \mathrm{d} \sqrt{p} .
$$

Similarly, pressure-dependent permeance can be defined using differential as shown in Table I. The flux can be estimated by

$$
J=\int_{p^{\mathrm{p}}}^{p^{\mathrm{f}}} P(p) \mathrm{d} p
$$

For a set of operating conditions, permeation fluxes in Eqs. (3) and (4) have to be the same. As a result, relation between pressure-dependent permeability and permeance is derived in the following form [10]:

$$
P(p)=\phi(p) /(2 l \sqrt{p}) .
$$

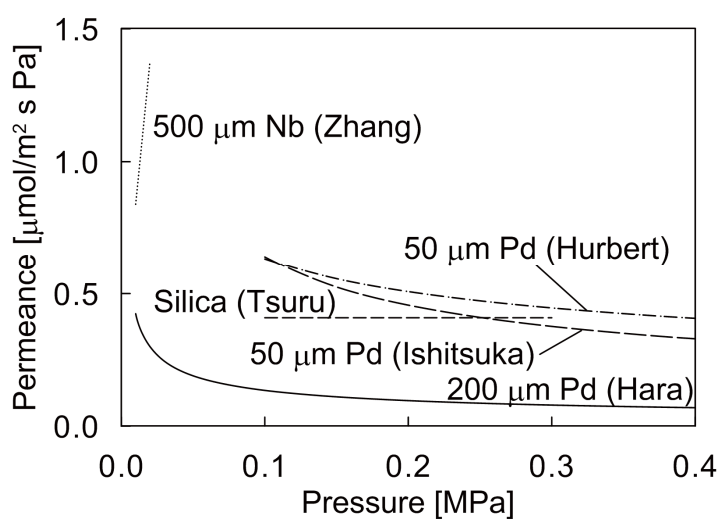

Fig. 1. Hydrogen permeance comparison among palladium, niobium, and silica membranes with different thicknesses.

\section{RESULTS AND DISCUSSION}

Pressure-dependent permeance at $773 \mathrm{~K}$ is collected in Fig. 1. Its derivation is described below. Discussion on Fig. 1 will be presented in the last section of this part.

\subsection{Palladium membrane $50 \mu \mathrm{m}$ thick}

Permeance of a pure palladium membrane $50 \mu \mathrm{m}$ thick is presented by Ishitsuka [10], drawn in Fig. 1. The membrane is a cold-rolled palladium foil purchased from Nilaco Co. (Japan). It was annealed at $973 \mathrm{~K}$ for 5 $\mathrm{h}$ in an $\mathrm{Ar}-5 \% \mathrm{H}_{2}$ atmosphere to reduce dislocations and point defects. Then hydrogen permeation was carried out in the range of $0.1-1.0 \mathrm{MPa}$. Therefore, permeance is drawn no less than $0.1 \mathrm{MPa}$ in the Figure.

\subsection{Palladium membrane $200 \mu \mathrm{m}$ thick}

To confirm the validity of pressure-dependent permeability under wider conditions, a thicker membrane was tested for a wider pressure range, i.e., $200 \mu \mathrm{m}$ thick and $0.01-1.0 \mathrm{MPa}$ [11]. The pure palladium sheet was cold rolled, purchased from Ishifuku Metal Industry Co., Ltd. (Japan). Permeation behavior was evaluated after annealing as well.

Hydrogen permeation flux was almost proportional to the square-root pressure difference, corresponding to the widely known square-root law. However, deviation from the proportionality was found. To evaluate pressuredependent permeability, the flux was fitted using third order of polynomials in terms of the square root of feed-side pressure for a constant permeation-side pressure. Differentiating the polynomials by the square

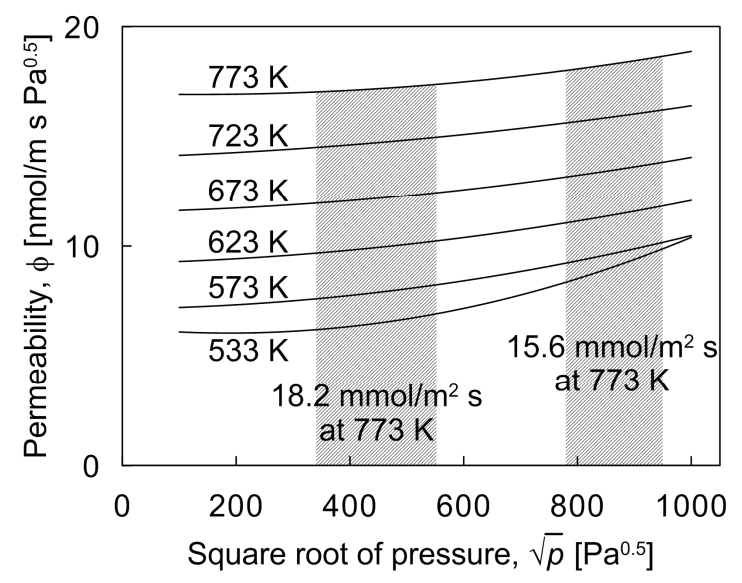

Fig. 2. Hydrogen permeability evaluated from a pure palladium membrane $200 \mu \mathrm{m}$ thick as a function of square root of pressure. Two gray areas respectively represent permeation flux values for different pressure conditions at $773 \mathrm{~K}$. 


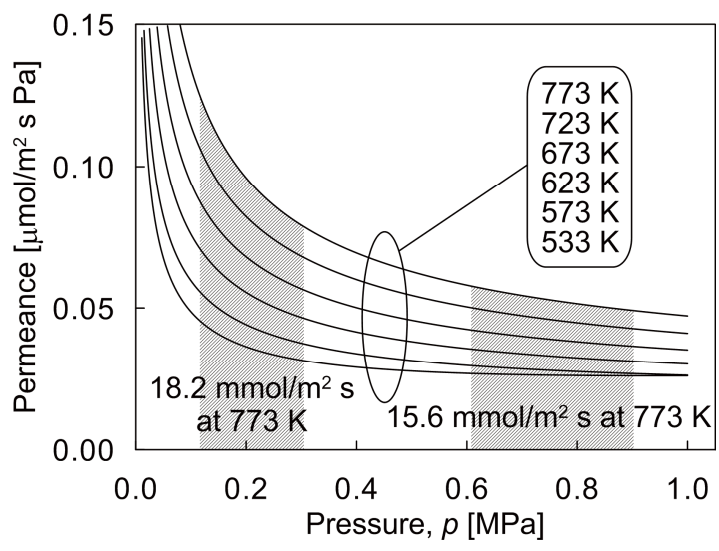

Fig. 3. Hydrogen permeance of a pure palladium membrane $200 \mu \mathrm{m}$ thick as a function of pressure. Two gray areas respectively represent permeation flux values for different pressure conditions at $773 \mathrm{~K}$.

root of feed-side pressure, pressure-dependent permeability was determined in the following form:

$$
\phi(p)=a(\sqrt{p})^{2}+b \sqrt{p}+c
$$

Parameters $a, b$, and $c$ were presented in the literature. For example, $a, b$, and $c$ for $773 \mathrm{~K}$ were $2.59 \times 10^{-15}$ $\mathrm{mol} / \mathrm{m} \mathrm{s} \mathrm{Pa}{ }^{1.5},-0.68 \times 10^{-12} \mathrm{~mol} / \mathrm{m} \mathrm{s} \mathrm{Pa}$, and $1.70 \times 10^{-8}$ $\mathrm{mol} / \mathrm{m}$ s $\mathrm{Pa}^{0.5}$, respectively [11]. Thus obtained permeability is drawn in Fig. 2. The permeability increases with pressure. The pressure dependency is the smaller at the lower pressures and the higher temperatures. These features are the same as those for the $50 \mu \mathrm{m}$ membrane reported previously [9].

According to Eq. (5), pressure-dependent permeance is given as follows:

$$
P(p)=\frac{1}{2 l}\left(a \sqrt{p}+b+\frac{c}{\sqrt{p}}\right) .
$$

The resultant permeance is illustrated in Fig. 3.

Pressure-dependent permeance can be determined directly using $P(p)_{p=p^{\mathrm{f}}}=\mathrm{d} J / \mathrm{d} p^{\mathrm{f}}$ in the Table I.

However, polynomials in terms of pressure itself instead of Eq. (7) are not useful to fit the experimental flux because the permeance differs largely from a linear form as seen in Fig. 3.

Permeation flux can be estimated by integrating Eqs. (3) and (4), corresponding to the gray areas in the Figures. Thus evaluated values are compared with the experiment and the conventional square-root law in Table II. Experimental permeation fluxes at $773 \mathrm{~K}$ from 0.305 to $0.116 \mathrm{MPa}$ and from 0.902 to $0.608 \mathrm{MPa}$ are 17.5 and $16.0 \mathrm{mmol} / \mathrm{m}^{2} \mathrm{~s}$, respectively. Because Eqs. (3) and (4) with pressure-dependent measures are mathematically equivalent, estimated fluxes by them are the same. They are 18.2 and $15.6 \mathrm{mmol} / \mathrm{m}^{2} \mathrm{~s}$, respectively, different from the experimental ones by $4.2 \%$ and $-2.5 \%$. In the conventional square-root law, permeability is determined as $1.71 \times 10^{-8} \mathrm{~mol} / \mathrm{m} \mathrm{s} \mathrm{Pa}^{0.5}$, resulting in 18.1 and $14.5 \mathrm{mmol} / \mathrm{m}^{2} \mathrm{~s}$. These values are different from the experiment by $3.4 \%$ and $-9.2 \%$, respectively. It is found that these pressure-dependent measures can estimate flux more precisely in a wide pressure range than the conventional constant measures.

3.3 Power law: Palladium membrane reported by Hurlbert and Konecny

As mentioned in Introduction, the power law is often adopted for precise description from 1901 [5]. The power law is represented by

$$
J=\frac{\phi^{\prime}}{l}\left\{\left(p^{\mathrm{f}}\right)^{n}-\left(p^{\mathrm{p}}\right)^{n}\right\}
$$

where $\phi^{\prime}$ and $n$ are fitting parameters, $\phi$ is permeability for the power law. Hurlbert and Konecny studied pure palladium membrane thicker than $20 \mu \mathrm{m}$ to determine $n$ $=0.68$ and $\phi^{\prime}=1.85 \times 10^{-9} \mathrm{~mol} / \mathrm{m} \mathrm{s} \mathrm{Pa}^{0.68}$ at $773 \mathrm{~K}[6]$. This power law's permeability depends on the power $n$, making it impossible to compare permeation ability using this measure.

Pressure-dependent measures enable comparison among the power law and other laws. Power law can be converted into pressure-dependent permeability by the following equation [9]:

$$
\phi(p)=2 n \phi^{\prime} p^{n-\frac{1}{2}} .
$$

Furthermore, permeance is derived using Eq. (5) as follows:

$$
P(p)=\frac{n \phi^{\prime} p^{n-1}}{l}
$$

The function is explicitly given as follows:

$$
P(p)=2.52 \times 10^{-5} p^{-0.32},
$$

which is drawn in Fig. 1.

Table II. Comparison of the fluxes estimated using the pressure-dependent measures and those in the conventional square-root law.

\begin{tabular}{lcc}
\hline & \multicolumn{2}{c}{$\begin{array}{c}\text { Permeation flux at } 773 \mathrm{~K} \text { through the palladium membrane } 200 \mu \mathrm{m}\left[\mathrm{mmol} / \mathrm{m}^{2} \mathrm{~s}\right] \\
\text { (Difference from the experimental flux) }\end{array}$} \\
\cline { 2 - 3 } & from 0.305 to $0.116 \mathrm{MPa}$ & from 0.902 to $0.608 \mathrm{MPa}$ \\
\hline $\begin{array}{l}\text { Experimental flux } \\
\begin{array}{l}\text { Pressure-dependent measures } \\
\text { (This study) }\end{array}\end{array}$ & 17.5 & 16.0 \\
$\begin{array}{l}\text { Square-root law } \\
\text { (Conventional for metal) }\end{array}$ & $18.2(+4.2 \%)$ & $15.6(-2.5 \%)$ \\
\hline
\end{tabular}


3.4 Niobium membrane

Zhang et al. studied a pure niobium membrane to find that the permeation flux did not obey Sieverts' law [7]. Therefore, they presented only permeation flux for a few operating conditions not permeability. Using pressure-dependent permeability, even such a behavior can be described precisely. The procedure is demonstrated in Ref. [9]. Because experimental values were not many, permeation flux was fitted by a second order polynomial in terms of the square-root of feed-side pressure. By differentiating it, pressure-dependent permeability was determined. Substituting the resultant permeability into Eq. (5), the following pressure-dependent permeance is derived:

$$
P(p)=2.68 \times 10^{-6}-\frac{1.84 \times 10^{-4}}{\sqrt{p}} .
$$

The resultant permeance increases with pressure differently from palladium membranes, as shown in Fig. 1. This unique behavior is attributable to great deviation from Sieverts' law. Pressure-dependent permeability and permeance are applicable to such a case.

\subsection{Porous silica membrane}

As an example other than metal membranes, a silica membrane is presented here. The membrane reported by Tsuru et al. had both a high permeation performance and a high selectivity to hydrogen against nitrogen, for example, $4.1 \times 10^{-7} \mathrm{~mol} / \mathrm{m}^{2} \mathrm{~s} \mathrm{~Pa}$ and 680 , respectively [8]. The permeance is drawn in Fig. 1 as a horizontal broken line.

Silica membranes have atomic scale pores, through which small molecule can pass without dissociating into hydrogen atoms. Therefore, permeation flux is fundamentally proportional to the pressure difference across the membrane without square root. For silica membranes, constant permeance is normally used.

\subsection{Comparison among membranes}

The pure niobium membrane has the highest permeance of all the membranes presented here, although it is the thickest. However, the niobium membrane is not applicable to large pressure difference. Therefore, when a wide pressure range is available, the $50 \mu \mathrm{m}$ palladium membranes or silica membrane becomes useful to obtain a high hydrogen flux. Permeance for palladium membranes increases with decreasing pressure. By comparing under the same pressure difference, low pressures are better, especially near the vacuum. On the other hand, the silica membrane is useful for high pressures because of the constant permeance.

Additionally, membranes with different thicknesses can be compared in the figure. For a small pressure difference, permeation flux of the $200 \mu \mathrm{m}$ palladium membranes around $0.01 \mathrm{MPa}$ is expected to be almost the same as that of the $50 \mu \mathrm{m}$ palladium membranes around $0.3 \mathrm{MPa}$.

Above discussion has become enabled for the fist time by this pressure-dependent permeance, useful to choose membranes suitable for each application as well as operating pressure conditions.

\section{CONCLUSIONS}

By introducing pressure-dependent permeance, membranes with different thicknesses and different materials were compared, including pure palladium membranes $50 \mu \mathrm{m}$ and $200 \mu \mathrm{m}$ thick, a pure niobium membrane, and a silica membrane without pressure dependence in permeance. The result demonstrates that the pressure-dependent measures can describe hydrogen permeation flux more precisely than conventional square-root law. Then, it enabled comparison among membranes with different thicknesses made of different materials. The comparison showed suitable operating pressure conditions for each membrane. For instance, palladium membranes were useful at low pressures whilst the silica membrane at high pressures if the pressure difference across the membrane is constant. This technique is expected to be a powerful tool in the membrane development in the future.

\section{References}

[1] J. Shu, B. P. A. Grandjean, A. Van Neste and S. Kaliaguine, Can. J. Chem. Eng., 69, 1036-1060 (1991).

[2] R. E. Buxbaum and T. L. Marker, J. Membr. Sci., 85, 29-38 (1993).

[3] M. D. Dolan, J. Membr. Sci., 362, 12-28 (2010).

[4] J. W. Phair and R. Donelson, Ind. Eng. Chem. Res., 45, 5657-5674 (2006).

[5] A. Winkelmann, Ann. Physik, 6, 104-115 (1901).

[6] R. C. Hurlbert and J. O. Konecny, J. Chem. Phys., 34, 655-658 (1961).

[7] G. X. Zhang, H. Yukawa, N. Watanabe, Y. Saito, H. Fukaya, M. Morinaga, T. Nambu and Y Matsumoto, Int. J. Hydrogen Energy, 33, 4419-4423 (2008)

[8] T. Tsuru, H. Shintani, T. Yoshioka and M. Asaeda, Appl. Catal. A, 302, 78-85 (2006).

[9] S. Hara, M. Ishitsuka, H. Suda, M. Mukaida and K. Haraya, J. Phys. Chem. B, 113, 9795-9801 (2009).

[10] M. Ishitsuka, S. Hara, H. Suda, M. Mukaida and K. Haraya, AMS 5 in Kobe (Conference proceedings), Kobe, 657-658 (2009).

[11] S. Hara, M. Ishitsuka, H. Suda, M. Mukaida and K. Haraya, Adv. Mater. Res., 117, 81-85 (2010).

(Received Janurary 17, 2011; Accepted February 16, 2011) 